\title{
Intellectual functioning and behavioural features associated with mosaicism in fragile $X$ syndrome
}

Emma K. Baker 1,2,3* (D), Marta Arpone 1,2,4, Solange Aliaga Vera', Lesley Bretherton ${ }^{4}$, Alexandra Ure ${ }^{2,5,6,7}$, Claudine M. Kraan ${ }^{1,2}$, Minh Bui ${ }^{8}$, Ling Ling ${ }^{1}$, David Francis ${ }^{9}$, Matthew F. Hunter ${ }^{7,10}$, Justine Elliott ${ }^{9}$, Carolyn Rogers ${ }^{11}$, Michael J. Field ${ }^{11}$, Jonathan Cohen ${ }^{12}$, Lorena Santa Maria ${ }^{13}$, Victor Faundes ${ }^{13}$, Bianca Curotto ${ }^{13}$, Paulina Morales ${ }^{13}$, Cesar Trigo $^{13}$, Isabel Salas ${ }^{13}$, Angelica M. Alliende ${ }^{13}$, David J. Amor ${ }^{2,5+}$ and David E. Godler ${ }^{1,2+}$

\begin{abstract}
Background: Fragile $X$ syndrome (FXS) is a common cause of intellectual disability and autism spectrum disorder (ASD) usually associated with a CGG expansion, termed full mutation (FM: CGG $\geq 200$ ), increased DNA methylation of the FMR1 promoter and silencing of the gene. Mosaicism for presence of cells with either methylated FM or smaller unmethylated pre-mutation (PM: CGG 55-199) alleles in the same individual have been associated with better cognitive functioning. This study compares age- and sex-matched FM-only and PM/FM mosaic individuals on intellectual functioning, ASD features and maladaptive behaviours.

Methods: This study comprised a large international cohort of 126 male and female participants with FXS (aged 1.15 to 43.17 years) separated into FM-only and PM/FM mosaic groups (90 males, 77.8\% FM-only; 36 females, 77.8\% FM-only). Intellectual functioning was assessed with age appropriate developmental or intelligence tests. The Autism Diagnostic Observation Schedule-2nd Edition was used to examine ASD features while the Aberrant Behavior Checklist-Community assessed maladaptive behaviours.

Results: Comparing males and females (FM-only + PM/FM mosaic), males had poorer intellectual functioning on all domains $(p<0.0001)$. Although females had less ASD features and less parent-reported maladaptive behaviours, these differences were no longer significant after controlling for intellectual functioning. Participants with PM/FM mosaicism, regardless of sex, presented with better intellectual functioning and less maladaptive behaviours compared with their age- and sex-matched FM-only counterparts $(p<0.05)$. ASD features were similar between FM-only and PM/FM mosaics within each sex, after controlling for overall intellectual functioning.
\end{abstract}

Conclusions: Males with FXS had significantly lower intellectual functioning than females with FXS. However, there were no significant differences in ASD features and maladaptive behaviours, after controlling for intellectual functioning, independent of the presence or absence of mosaicism. This suggests that interventions that primarily target cognitive abilities may in turn reduce the severity of maladaptive behaviours including ASD features in FXS.

Keywords: Fragile X syndrome, Mosaicism, Autism spectrum disorder, Intellectual functioning, Behaviour, Phenotype

\footnotetext{
* Correspondence: emma.baker@mcri.edu.au

${ }^{\dagger}$ David J. Amor and David E. Godler contributed equally to this work.

'Diagnosis and Development, Murdoch Children's Research Institute, Royal

Children's Hospital, Melbourne, VIC, Australia

${ }^{2}$ Faculty of Medicine, Dentistry and Health Sciences, Department of

Paediatrics, University of Melbourne, Parkville, VIC, Australia

Full list of author information is available at the end of the article
}

(c) The Author(s). 2019 Open Access This article is distributed under the terms of the Creative Commons Attribution 4.0 International License (http://creativecommons.org/licenses/by/4.0/), which permits unrestricted use, distribution, and reproduction in any medium, provided you give appropriate credit to the original author(s) and the source, provide a link to the Creative Commons license, and indicate if changes were made. The Creative Commons Public Domain Dedication waiver (http://creativecommons.org/publicdomain/zero/1.0/) applies to the data made available in this article, unless otherwise stated. 


\section{Background}

Fragile X syndrome (FXS) is a common single-gene cause of inherited intellectual disability (ID) and autism spectrum disorder (ASD), with current prevalence estimates of 1:4000 and 1:8000 males and females, respectively [1]. The clinical phenotype of FXS is heterogeneous, affected by age, sex and genetic and epigenetic factors (e.g. $\mathrm{X}$ chromosome inactivation in females). The primary cause of FXS is a large trinucleotide CGG expansion ( $\geq$ 200 repeats), termed full mutation (FM), in the promoter region of the fragile $\mathrm{X}$ mental retardation 1 (FMR1) gene [2]. FM alleles are associated with DNA methylation (DNAm) changes to the FMR1 promoter, resulting in decreased transcription [3] and little to no production of the fragile X mental retardation protein (FMRP). FMRP has been shown to regulate genes that have been implicated in ASD [4] and to be important for neurodevelopment [5].

Mosaicism for smaller alleles usually unmethylated, termed pre-mutation (PM 55-199 CGG repeats) in combination with methylated FM alleles has been reported in $34 \%$ of males and $10 \%$ of females with FXS using methylation-sensitive Southern blot techniques $[6,7]$. The phenotype is typically reported to be attenuated in such cases due to the presence of cells with the unmethylated FMR1 promoter, capable of producing FMRP, though variability is still observed [8]. Nonetheless, the biological mechanisms responsible for the variability in the FXS phenotype especially in females and $\mathrm{PM} / \mathrm{FM}$ mosaic individuals is not well understood. The contribution of genotype mosaicism on psychological functioning, within and between sexes, may shed light on the underlying biology that contributes towards the cognitive, emotional and behavioural phenotype in males and females with FXS.

Females with FM are typically reported to be less affected than males due to the presence of the second normal size $(<44$ CGG) FMR1 allele located on the active $\mathrm{X}$ chromosome in some cells [9]. The vast majority of males with FXS typically present with ID $[7,8,10]$ with intellectual quotients (IQs) below 70. Their clinical profile is also characterised by attention and executive functioning difficulties, anxiety, social withdrawal, hyperactivity, impulsivity and ASD features [11]. The phenotype in females with a FM is less predictable, which in part may be due to an underrepresentation of females in the FXS literature [12]. A wide range of intellectual abilities has been associated with the female FXS phenotype, with IQ ranging from the extremely low to the superior range $[13,14]$. Females with FXS can also present with a variety of behavioural and emotional problems, including attention difficulties, anxiety, withdrawn behaviours and ASD features with associated impacts on outcomes $[12,15,16]$. In particular, elevated rates of socially avoidant behaviours and repetitive and restricted behaviours (RRB) have been noted in females with FXS compared with non-FXS females [17, 18].

Males with mosaic FXS have been generally reported to have better intellectual functioning than their FMonly counterparts [19]. However, studies comparing rates of ASD between FM-only and mosaic cases are somewhat limited, as these have primarily focused on males, not both sexes. A study of boys with FXS (mean age $56.6 \pm 13.7$ months) demonstrated that 18 of 41 (43.9\%) participants with FM and 6 of 15 (40\%) participants with mosaicism (DNAm + PM/FM) met DSM-IV criteria for ASD [20]. In contrast, in an earlier study [21], there was a trend for ASD to be more likely diagnosed in FM-only males $(44 \% ; n=18)$ in comparison with males with mosaic FXS $(18 \% ; n=28)$.

In females, Rousseau and colleagues [8] did not identify a significant difference in the distribution of degrees of intellectual impairment between females with FMonly and PM/FM mosaicism. Remarkably, intellectual functioning was not formally assessed and was assigned based on clinical judgement. Thus, further research comparing intellectual functioning and behavioural features between females with FM-only and PM/FM mosaicism with validated standardised measures is an important gap that needs to be addressed.

Chronological age has also been associated with the clinical phenotype, with an apparent cognitive decline in males with FXS. However, a more recent large prospective longitudinal study [14] identified a widening gap in cognitive performance between participants with FXS relative to the normative sample in childhood, but with a stabilisation or narrowing of this gap during adolescence. For behavioural profiles, increasing chronological age has been associated with decreases in scores on the Aberrant Behavior Checklist-Community (ABC-C [22]) in both males and females with FXS [23]. While ASD prevalence and symptom severity was reported to increase over time in individuals with FXS [24].

One of the most consistently reported behavioural characteristics of FXS is the presence of ASD features, with up to $90 \%$ of males and $50 \%$ of females demonstrating some symptoms associated with ASD [11, 25]. However, there is contention as to whether comorbid ASD in FXS is a true phenomenon, or indeed that ASD and FXS represent categorically and qualitatively distinct disorders [26, 27]. Reported prevalence of ASD in FXS varies between studies depending on the assessment tools and diagnostic criteria that are used. In a systematic review and meta-analysis of ASD phenomenology in various genetic disorders, approximately $22 \%$ of individuals with FXS met criteria for ASD [28]. The prevalence estimate rose to $30 \%$ when only males with FXS were included. The presence of seizures in FXS has also been linked to the presence of ASD. Utilising the fragile $\mathrm{X}$ clinical and 
research consortium database prevalence rates for seizures in males and females with FXS are $12.1 \%$ and $3.2 \%$, respectively, with estimates for males and females with FXS + ASD being 16\% [29].

The differences in prevalence estimates of ASD observed between males and females with FXS have been attributed to the more favourable genetic and biochemical functioning of females (i.e. the presence of a normal FMR1 allele on the active $\mathrm{X}$ chromosome expressing FMRP) [27]. Moreover, the more severe intellectual impairments in males with FXS have been associated with more ASD features [27]. Furthermore, idiopathic ASD is more common in males [30, 31]. Several biological explanations for the male preponderance in ASD have been proposed, including male vulnerability, female protection and the possibility of ascertainment bias and associated female under diagnosis (see Ferri et al. [30] for a review) particularly for those without an ID [32]. To this end, Ratto and colleagues [33] undertook itemlevel analysis on the ADOS-2 to determine the specific profiles of males and females on this measure. The authors demonstrated that while IQ-matched males and females had similar overall calibrated severity scores (CSS) and that item-level differences did indeed emerge, none of these differences survived corrections for multiple testing. In contrast, similar analyses comparing males and females with FXS have not previously been undertaken.

FMRP levels in blood have been shown to be associated with developmental and intellectual functioning levels in males with FXS, with lower levels of FMRP corresponding to more severe cognitive impairments $[10,34,35]$. However, similar relationships have not been shown with behavioural symptoms [36, 37]. Specifically, FMRP levels in peripheral tissues (that may not accurately reflect brain specific variability in FMRP) were not associated with parent reports of behavioural problems, adaptive behaviours or ASD features in a large sample of male and female children (6 to 17 years) with FXS [36]. Moreover, in studies using objective assessments including the Autism Diagnostic Observation Schedule-Generic (ADOS-G), the associations between FMRP and ASD symptom severity were lost after controlling for IQ [37]. Similarly, McDuffie and colleagues found that FMRP did not account for unique variance in reciprocal social interaction, communication and restricted interests, and repetitive behavior domain scores on the Autism Diagnostic Interview-Revised (ADI-R) [38], over and above the contribution of nonverbal IQ, though this study included both males and females in the same cohort, allowing greater data dispersion. More recently, FM-only males with incomplete silencing of FMR1 mRNA have been reported to have significantly greater severity of ASD features (ADOS-2 calibrated severity scores [CSS]) compared with FM-only males with complete silencing. This suggests that two reciprocal mechanisms, RNA toxicity and FMRP deficiency, may contribute to ID and ASD features in those males with FXS and the FMR1 gene incompletely silenced [39], though replication of this study in larger independent cohorts is required.

Female-only studies are few, with inconsistent findings between FMRP levels and the clinical phenotype. Some studies have demonstrated associations with intellectual functioning $[35,40]$ and the behavioural phenotype [41, 42], while others have demonstrated no associations between these constructs and FMRP levels in females with FXS [26, 41, 43-45]. Inconsistent findings in females may be due to the age ranges used in each of the studies. Specifically, Lightbody and colleagues [45] demonstrated that chronological age was associated with executive functioning abilities in females with FXS, but not FMRP levels in blood.

While previous research has comprehensively documented the intellectual functioning phenotypes in males and females with FXS, and to some extent PM/FM mosaic males, there is little understanding of how ASD features and maladaptive behaviours differ in males and females with FXS, as well as between sex-matched FMonly and PM/FM mosaic individuals. Understanding predictors of the behavioural phenotype in both males and females with FXS will assist in determining potential targets for interventions that can assist in improving outcomes. Thus, this study characterises a large international FXS cohort in order to: (i) describe and compare IQ, ASD features and maladaptive behaviours between sexes and between allelic classes within each sex; and (ii) evaluate if the variability of behavioural problems within each sex and between allelic classes is explained by the variability in intellectual functioning.

\section{Methods \\ Participants}

This study used a large international FXS cohort recruited from Australia and Chile. Ninety males (51.1\% Australian) and 36 females (75\% Australian) participated in the study. Male participants were aged between 1.15 and 43.17 years $(M=12.06, \mathrm{SD}=9.82)$ while female participants were aged between 1.71 and 34.13 years $(M=10.90, \mathrm{SD}=9.23)$. The cohort of individuals encompasses a wide age range to enable a sufficient number of females to be included in the current study. In the male cohort, $70(77.8 \%)$ participants were FM-only and $20(22.2 \%)$ were PM/FM mosaic. In the female cohort, 28 (77.8\%) were FM-only and 8 (22.2\%) were PM/FM mosaic.

Australian participants were recruited via Victorian Clinical Genetics Services, Monash Genetics and Hunter 
Genetics, and support organisations including the Fragile $\mathrm{X}$ Association of Australia and Fragile X Alliance Inc. In Chile, participants were recruited primarily through Molecular and Cytogenetics at INTA, University of Chile. Individuals were excluded from the study if they had any other significant medical conditions (e.g. stroke, head trauma), any other genetic conditions of known clinical significance, and if they had inadequately controlled seizures. Parents completed a developmental and medical history questionnaire to obtain information regarding ethnicity, medication use and other previous or existing diagnoses (e.g. lifetime prevalence of seizures).

\section{Materials}

\section{Genetic testing}

For the Chilean and Australian participants, inclusion criteria into this study included a confirmed diagnosis of FXS using methylation SB and CGG PCR sizing. Specifically, for all participants, routine FXS testing involved first-line PCR-based assessment of CGG repeat size (with precision of \pm one repeat) using a validated PCR amplification assay, with the upper limit of detection of 330 CGG repeats for the Chilean cohort [46] and 170 CGG repeats for the Australian cohort [47]. All samples that showed a CGG size in the PM range or that failed to show a PCR product for males or had a single peak by PCR for females were referred for second-line testing involving methylation-sensitive SB analysis, performed as described $[48,49]$. If there were any discrepancies between SB performed between the Australian and Chilean sites, AmplideX long-range PCR was undertaken, as per manufacturer's instructions [50] on blood or saliva DNA collected as previously described [49]. Original diagnostic reports were also obtained for all Australian participants to confirm diagnosis.

\section{Assessment of intellectual functioning}

Participants were assessed with one of the following standardised assessments depending on their age and country of residence: the Mullen Scales of Early Learning (MSEL; Australian children aged < 3 years) [51], the Wechsler Preschool and Primary Scale of Intelligence3rd Australian [52] and Mexican [53] Editions (WPPSIIII; children aged 3-6 years), the Wechsler Scale of Intelligence-4th Edition Australian (WISC-IV; Australian children aged 7-16 years) [54], Wechsler Scale of Intelligence-3rd Edition Chilean version [55] (WISC-III; Chilean children aged 7-16 years) or the Wechsler Adult Intelligence Scale-4th Australian [56] and Chilean [57] Editions (WAIS-IV; $\geq 17$ years). As the MSEL does not provide separate verbal IQ (VIQ) and performance IQ (PIQ) scores, these were derived as described by Richler and colleagues [58]. The Early Learning Composite (ELC) was used as a proxy for Full Scale IQ (FSIQ) [59]. The breakdown of which intellectual functioning assessments were completed, by sex and allelic classification, is presented in Additional file 1: Table S1 (see also Additional file 1: Note S1).

Given the tendency for individuals with FXS, particularly males, to have scores on standardised assessments at floor level, we used corrected IQ scores [60]. Corrected IQ scores were derived using the Whitaker and Gordon method [61]. Briefly, all scaled scores (SS) of 1 were re-examined. In order to calculate corrected index scores (e.g. cVIQ, cFSIQ), the best fit equations between raw scores and SS, and subsequently between sum of scaled scores (SSS) and index scores were determined by using the raw score to SS and the SSS to index scores conversion tables available in the published test manuals. For an example of the application of this technique, see the supplemental material of Arpone et al. [60]. This method reduces loss of data due to invalid scores and has been shown to result in a normal distribution of scores [60]. A caveat to this method in the current sample is that the WISC-III (Chilean version) incorporates more sub-tests in the verbal and performance indexes than the other Wechsler scales used in this study. Subtests that are included in the working memory and processing speed indices of the other Wechsler scales (i.e. WISC-IV and WAIS-IV) are included in the VIQ and PIQ, respectively, of the WISC-III. Moreover, while VIQ, PIQ and FSIQ scores can be derived from the MSEL, the assessment is qualitatively different to the Wechsler scales and correction of these scores has not previously been undertaken. Therefore, in the supplementary materials, we also present the analyses using the following: (i) standard scores and (ii) corrected scores with participants assessed with the MSEL and WISC-III removed.

\section{Assessment of autism spectrum disorder features}

The Autism Diagnostic Observation Schedule-2nd Edition (ADOS-2) [62] was used to assess ASD features. Participants are assessed with one of five modules depending on their age and language abilities. For modules $1-4$, the ADOS-2 provides three specific classifications: non-spectrum, autism spectrum and autism. For the toddler module, classifications are based on range of concern: little-to-none, mild-to-moderate and moderateto-severe. Those falling in the little-to-none were classified as non-spectrum, while those in the latter two ranges were classified as autism spectrum and autism, respectively. Separate CSS based on the overall, social affect (SA) and restricted and repetitive behaviour (RRB) scores are also derived for each module [63-65]. The breakdown of modules completed, by sex and allelic classification, is presented in Additional file 1: Table S1. ADOS-2 assessments and coding were conducted by research members who had undertaken ADOS-2 for 
research training and had demonstrated $>80 \%$ coding reliability across all five modules. ADOS- 2 assessments were video recorded, with consent, and a proportion were re-coded with adequate reliability (see also Additional file 1: Note S2).

\section{Assessment of maladaptive behaviours}

Maladaptive behaviours were assessed in both cohorts using the Aberrant Behavior Checklist-Community $(\mathrm{ABC}-\mathrm{C})$ [22]. The $\mathrm{ABC}-\mathrm{C}$ is a parent-report questionnaire that is commonly used to assess maladaptive behaviours in children with ID. In contrast to the original ABC-C, Sansone and colleagues [66] found a six-factor structure when used in a sample of 630 individuals with FXS. The inappropriate speech domain remained unchanged while the remaining four subscales were modified. A new social avoidance subscale was derived for the sixth factor. These new subscales have demonstrated good internal consistency previously (Cronbach's $\alpha=$ .80-.94 [66]) and in the current sample (Cronbach's $\alpha=$ .82-.94). Kerr et al. [67] also developed a utility index (UI) to determine FXS quality of life based on the $\mathrm{ABC}$ C. Higher scores on the $\mathrm{ABC}-\mathrm{C}$ indicate greater maladaptive behaviours, while lower UI scores indicate lower/poorer quality of life. Two PM/FM mosaic adult females attended their appointment without a parent/ carer and therefore, no $\mathrm{ABC}-\mathrm{C}$ was available for these participants (see Additional file 1: Note S3).

\section{Procedure}

Participants were initially screened for eligibility criteria. Eligible participants were booked for assessment by a member of the research team that would not be involved in assessments, to ensure the allelic classification was blinded to the assessor. In the majority of cases, the ADOS-2 was administered first, followed by the intellectual functioning assessment. Assessments were typically undertaken on the same day by the same assessor. Two participants were assessed over 2 days to alleviate their distress. A third participant was also assessed over 2 days to accommodate a multiplex family where three children were assessed in succession over a 2-day period. In Chile, all assessments were undertaken in the clinic, while in Australia, $75.3 \%$ of assessments were completed in a clinic; the remaining assessments were undertaken in the home.

\section{Statistical analysis}

Summary statistics were presented as frequencies and proportion for categorical variables and mean and standard deviation (SD) for continuous variables. Comparisons were performed between: (i) males and females, and (ii) FM-only and PM/FM mosaic groups for both sexes. Comparisons for categorical variables (demographic information and key
ADOS-2 items) were carried out using Fisher's exact test. For continuous variables, comparisons were performed using regression methods, including a between-group term, adjusted for potential confounders, age and country. A non-linear relationship between each corrected intellectual functioning score and age was observed only in males, and therefore, we used semi-parametric regression (see Additional file 1: Note S4 for further details). Additionally, there were significant differences between the two countries on corrected intellectual functioning variables for males, but not females. For females, no significant relationship was observed between corrected intellectual functioning scores and age. Given the wide age range included in the study, where possible analyses were also conducted in stratified age groups, under 13 years and 13 years and over. Details of the specific analyses including which confounders were controlled in each analysis are described in the footnotes of each table.

Comparisons between males and females and allelic classes on key ADOS-2 items were performed by exploring the proportion of individuals who had atypical presentations on each item. Codes of 1,2 and 3 were combined to indicate an atypical presentation on the specific behaviour, while scores of 0 indicated a typical presentation.

False discovery rate (FDR) was used to adjust for multiple testing. All analyses were carried out using commercial software Stata version 15 (http://www.stata.com), and $p$ values were two-sided with an alpha level of 0.05 .

\section{Results \\ Medical history and demographic information comparisons}

There were no differences in the parent-reported lifetime prevalence of seizures and ethnicity between FM and PM/FM mosaics within each sex and between sexes (Table 1). Males were more likely to be taking psychoactive mediations compared with females, though medication use did not differ between FM-only and PM/FM mosaic cases within each sex. The majority of individuals reported to have ever experienced seizures, met criteria for ASD on the ADOS- 2 and also met criteria for ID (FSIQ < 70; 8 males with FM-only, 1 male with PM/FM mosaicism, 1 female with FM-only). There was only one individual (FM-only male) with parent-reported seizures who did not meet criteria for ASD. The two sexes did not differ on age $(p=0.541)$.

\section{Correlation between ASD features and corrected intellectual functioning}

Corrected VIQ and PIQ (cVIQ and cPIQ) were inversely correlated with ASD features (SA CSS and RRB CSS), in males and females (FM-only + PM/FM mosaic), with the exception of cPIQ and SA CSS in females, and cVIQ 
Table 1 Demographic information for males and females by allelic classes

\begin{tabular}{|c|c|c|c|c|c|c|c|}
\hline & \multicolumn{3}{|l|}{ Males } & \multicolumn{3}{|l|}{ Females } & \multirow{2}{*}{$\begin{array}{l}\text { Males vs } \\
\text { females } \\
p\end{array}$} \\
\hline & $\begin{array}{l}\text { FM-only } \\
(n=70)(\%)\end{array}$ & $\begin{array}{l}\text { PM/FM mosaic } \\
(n=20)(\%)\end{array}$ & $p$ & $\begin{array}{l}\text { FM-only } \\
(n=28)^{a}(\%)\end{array}$ & $\begin{array}{l}\text { PM/FM mosaic } \\
(n=8)(\%)\end{array}$ & $p$ & \\
\hline Seizures $^{b}$ & 13.0 & 5.6 & 0.680 & 3.7 & 0.0 & 0.999 & 0.175 \\
\hline \multicolumn{8}{|l|}{ Medication } \\
\hline Medicated & 44.3 & 30.0 & 0.309 & 25.9 & 0.0 & 0.166 & 0.036 \\
\hline Stimulant & 20.0 & 10.0 & 0.508 & 7.4 & 0.0 & 0.999 & 0.097 \\
\hline SSRI & 21.4 & 5.0 & 0.109 & 11.1 & 0.0 & 0.999 & 0.271 \\
\hline SNRI & 0.0 & 5.0 & 0.222 & 3.7 & 0.0 & 0.999 & 0.483 \\
\hline Benzodiazepines & 0.0 & 5.0 & 0.222 & 0.0 & 0.0 & - & 0.999 \\
\hline Anti-psychotic & 11.4 & 10.0 & 0.999 & 3.7 & 0.0 & 0.999 & 0.180 \\
\hline Anticonvulsant & 7.1 & 5.0 & 0.999 & 3.7 & 0.0 & 0.999 & 0.672 \\
\hline $\begin{array}{l}\text { Adrenergic alpha } \\
\text { agonist }\end{array}$ & 4.3 & 5.0 & 0.999 & 3.7 & 0.0 & 0.999 & 0.999 \\
\hline Melatonin & 8.6 & 0.0 & 0.333 & 0.0 & 0.0 & 0.999 & 0.184 \\
\hline \multicolumn{8}{|l|}{ Ethnicity $^{c}$} \\
\hline Australian/European & 36.2 & 55.0 & 0.407 & 66.7 & 50.0 & 0.219 & 0.076 \\
\hline Mestizo & 46.4 & 35.0 & & 18.5 & 50.0 & & \\
\hline Other* & 17.4 & 10.0 & & 14.8 & 0.0 & & \\
\hline
\end{tabular}

${ }^{a}$ Demographic information was not available for one female with FM-only

${ }^{b}$ Seizure information was missing for 2 males with FM-only and 1 male with PM/FM mosaicism

${ }^{\mathrm{C} E t h i n i c t y ~ w a s ~ m i s s i n g ~ f o r ~ o n e ~ m a l e ~ w i t h ~ F M-o n l y ~}$

*Other ethnicity included Australian Aboriginal, Asian (Southern/South East), Middle Eastern, Polynesian, European (southern/northwest), American Indian, multi-

ethnic and not disclosed. All $p$ values computed using Fisher's exact test

and RRB CSS in males (Additional file 1: Table S3). The ADOS CSS was also inversely correlated with all corrected intellectual functioning scores in males and females (all $p<0.05$ ), except between cWMI in females $(p=0.253)$. Thus, when comparing corrected IQ scores analyses were adjusted for ADOS CSS and similarly when comparing ADOS CSS, analyses were adjusted for corrected IQ scores.

\section{Comparison of intellectual functioning, ASD features and maladaptive behaviours between males and females}

Sixty-three percent of males and $7.1 \%$ of females with FXS obtained a standard FSIQ score that was either invalid or at floor level (e.g. FSIQ $=40$ ). The proportions of standard IQ scores that were invalid and at floor level, stratified by sex and allelic class, are reported in Additional file 1: Table S2. A significantly higher proportion of males $(93.3 \%)$ had an ID (standard FSIQ $<70$ ), compared with females (47.2\%; Fisher's exact test, $p<0.001$ ). Males with FXS had significantly poorer corrected IQ scores on all domains compared with females (Table 2). These significant differences were still observed when standard scores were used and when the MSEL and WISC-III scores were removed from the corrected scores (Additional file 1: Tables S4 and S5, respectively).
A significantly higher proportion of males $(90.4 \%)$ met ADOS-2 criteria for ASD compared with females (65.6\%; Fisher's exact test, $p=0.004)$. Examining the specific ADOS-2 cutoffs 13 (40.6\%) females and 19 males (22.9\%) met the autism spectrum/mild-to-moderate concern cutoff, while eight (25.0\%) females and 56 (67.5\%) males met the autism/moderate-to-severe concern cutoff. However, males and females did not differ significantly on ADOS-2 CSS scores after controlling for country and cFSIQ (Table 2).

Moreover, when the data was stratified according to two age groups ( $<13$ years and $\geq 13$ years), the differences between males and females for corrected IQ scores, ASD features and maladaptive behaviours were similar to those shown in Table 2 (Additional file 1: Tables S6 and S7).

Comparisons between males and females on specific ADOS-2 items showed significant differences on several items including eye contact, shared enjoyment, showing, initiation of joint attention, rapport, sensory behaviours, response to name, amount of social overtures (both to the examiner and caregiver), amount of reciprocal communication and functional and creative play items (Table 3). Similar results were observed for individuals aged $<13$ years (Additional file 1: Table S8). However, for the older age group ( $\geq 13$ years), no significant differences between males and females were observed, likely 
Table 2 Comparison between males and females on corrected intellectual functioning scores, ASD features, and maladaptive behaviours

$\begin{array}{lll}\frac{\text { Males }}{n \quad \text { Mean } \pm S D} & \text { Females } & p \\ n & \text { Mean } \pm S D & \end{array}$

Intellectual functioning

\begin{tabular}{|c|c|c|c|c|c|}
\hline cVIQ & 87 & $45.3 \pm 23.6$ & 36 & $74.1 \pm 18.6$ & $<0.0001^{*}$ \\
\hline CPIQ & 88 & $44.7 \pm 19.7$ & 36 & $69.0 \pm 16.9$ & $<0.0001^{*}$ \\
\hline cWMI & 34 & $35.8 \pm 15.1$ & 13 & $66.0 \pm 18.6$ & $<0.0001^{*}$ \\
\hline CPSI & 46 & $45.1 \pm 17.6$ & 22 & $80.1 \pm 20.0$ & $<0.0001^{*}$ \\
\hline CFSIQ & 87 & $33.3 \pm 24.2$ & 36 & $67.8 \pm 17.8$ & $<0.0001^{*}$ \\
\hline \multicolumn{6}{|l|}{ ASD Features $^{2}$} \\
\hline ADOS CSS & 80 & $6.54 \pm 2.11$ & 32 & $4.53 \pm 2.29$ & 0.090 \\
\hline SA CSS & 80 & $6.24 \pm 2.25$ & 32 & $4.56 \pm 2.15$ & 0.253 \\
\hline RRB CSS & 80 & $7.58 \pm 1.89$ & 32 & $6.16 \pm 2.64$ & 0.129 \\
\hline \multicolumn{6}{|l|}{ Maladaptive behaviours ${ }^{3}$} \\
\hline Irritability & 87 & $14.1 \pm 11.0$ & 34 & $10.1 \pm 11.3$ & 0.523 \\
\hline Lethargy & 87 & $6.22 \pm 5.20$ & 34 & $4.62 \pm 5.41$ & 0.829 \\
\hline Stereotypy & 87 & $5.22 \pm 4.66$ & 34 & $2.76 \pm 4.31$ & 0.057 \\
\hline Hyperactivity & 87 & $11.3 \pm 8.23$ & 34 & $6.79 \pm 7.50$ & 0.071 \\
\hline Inappropriate speech & 87 & $4.45 \pm 3.51$ & 34 & $2.59 \pm 2.96$ & 0.724 \\
\hline Social avoidance & 87 & $3.02 \pm 2.78$ & 34 & $2.38 \pm 3.21$ & 0.130 \\
\hline$A B C$ total & 87 & $44.3 \pm 28.0$ & 34 & $29.2 \pm 29.3$ & 0.306 \\
\hline$A B C \cup I$ & 87 & $0.64 \pm 0.19$ & 34 & $0.73 \pm 0.20$ & 0.415 \\
\hline
\end{tabular}

${ }^{1}$ Semi-parametric regression adjusted for country, age and ADOS CSS

${ }^{2}$ Robust regression adjusted for country and CFSIQ ${ }^{3}$ Robust Regression adjusted for CFSIQ and age

${ }^{*} p$ value remained $<0.05$ after adjustment for multiple testing using FDR

due to the small sample of females $(n=8)$ in this age group reducing the power to detect significant differences (Additional file 1: Table S9).

Males and females did not significantly differ on any ABC-C subscales after adjusting for age and cFSIQ (Table 2). When comparing the specific classifications of the UI, a significantly greater proportion of males $(47.8 \%)$ fell in the very low or low range compared with females (23.5\%), $p=0.008$. Nineteen (55.9\%) females were classified as having high or very high quality of life compared with only 24 (26.7\%) males. All other individuals fell in the moderate range.

\section{Comparison of intellectual functioning, ASD features and maladaptive behaviours between males with FM-only and PM/FM mosaicism}

Sixty-five (94.2\%) males with FM-only and 18 (90.0\%) males with PM/FM mosaicism met criteria for ID ( $p=$ 0.613). However, males with FM-only had statistically significant lower cVIQ, cPSI and cFSIQ compared with males with PM/FM mosaicism (Table 4), though the difference for cVIQ did not survive FDR. When standard IQ scores were used VIQ, WMI and PSI differed significantly between the two groups (Additional file 1: Table S10). When those males assessed with the MSEL and WISC-III were removed from the corrected scores, differences between the male PM/FM mosaic and FMonly groups were no longer significant (Additional file 1: Table S11). When stratified by age, significant differences were observed for males under 13 years for all intellectual functioning scores, except cPIQ after adjusting for multiple testing (Additional file 1: Table S12). However, no significant differences were observed between males with FM-only and PM/FM mosaicism aged 13 years and above (Additional file 1: Table S13).

There were no significant differences between the proportion of participants with FM (89.2\%) and PM/FM mosaicism (94.4\%) who met criteria for ASD $(p=0.668)$. The two groups also did not significantly differ on each of the ADOS-2 CSS, both prior to and after adjusting for cFSIQ (Table 4). Similar results for ADOS-2 CSS were observed for both stratified age groups (Additional file 1: Tables S12 and S13).

The PM/FM mosaic group had significantly lower scores on the irritability, inappropriate speech and social avoidance subscales of the ABC-C compared with the FM-only group, after controlling for age. The PM/FM mosaic group also had lower total $\mathrm{ABC}-\mathrm{C}$ scores and a higher UI (Table 4). No other significant differences were observed between the two groups on the ABC-C. The proportion of males falling into each of the UI categories did not significantly differ between males with FM-only and males with PM/FM mosaicism (Fisher's exact test, $p=0.292$ ). When stratified by age, no significant differences were observed for males under 13 years after adjusting for multiple testing (Additional file 1: Table S12). However, for those males aged 13 and over, all $\mathrm{ABC}-\mathrm{C}$ domains significantly differed between the PM/FM mosaic and FM-only groups, except hyperactivity (Additional file 1: Table S13).

\section{Comparison of intellectual functioning, ASD features and maladaptive behaviours between females with FM-only and PM/FM mosaicism}

A significantly greater proportion of females with FMonly had FSIQ $<70(57.1 \%)$ compared with females with PM/FM mosaicism (12.5\%; $p=0.044)$. Females with PM/FM mosaicism had higher scores on all corrected intellectual functioning domains compared with females with FM-only (Table 5). These same findings were shown when using standard IQ scores and when the MSEL and WISC-III were removed from corrected scores (Additional file 1: Table S14 and S15, respectively).

Seventeen (70.8\%) females with FM-only met criteria for ASD on the ADOS-2 compared with four (50\%) in the $\mathrm{PM} / \mathrm{FM}$ mosaic group. However, this difference was not significant (Fisher's exact test, $p=0.397$ ). Furthermore, 
Table 3 Comparison between males and females on key ADOS-2 items

\begin{tabular}{|c|c|c|c|c|c|}
\hline & \multicolumn{2}{|c|}{ Males } & \multicolumn{2}{|c|}{ Females } & \multirow[t]{2}{*}{$p$} \\
\hline & $n$ & $\%$ atypical & $n$ & $\%$ atypical & \\
\hline \multicolumn{6}{|l|}{ SA } \\
\hline Pointing & 62 & 71.0 & 17 & 76.5 & 0.767 \\
\hline Gestures & 83 & 55.4 & 32 & 37.5 & 0.099 \\
\hline Eye contact & 83 & 81.9 & 32 & 53.1 & $0.004^{*}$ \\
\hline Facial expressions & 83 & 88.0 & 32 & 71.9 & 0.050 \\
\hline Shared enjoyment & 83 & 49.4 & 32 & 18.8 & $0.003^{*}$ \\
\hline Showing & 62 & 77.4 & 17 & 35.3 & $0.002^{*}$ \\
\hline Response to joint attention & 62 & 29.0 & 17 & 11.8 & 0.212 \\
\hline Initiation of joint attention & 62 & 71.0 & 17 & 29.4 & $0.004^{*}$ \\
\hline Quality of social overtures & 83 & 88.0 & 32 & 75.0 & 0.095 \\
\hline Rapport & 83 & 72.3 & 32 & 46.9 & $0.016^{*}$ \\
\hline \multicolumn{6}{|l|}{ RRB } \\
\hline Sensory & 83 & 62.7 & 32 & 31.3 & $0.003^{*}$ \\
\hline Mannerisms & 83 & 59.0 & 32 & 46.9 & 0.296 \\
\hline Repetitive and stereotyped behaviours & 83 & 73.5 & 32 & 68.8 & 0.646 \\
\hline Stereotyped language & 62 & 78.7 & 27 & 63.0 & 0.187 \\
\hline \multicolumn{6}{|l|}{ Other } \\
\hline Use of body as tool & 39 & 38.5 & 9 & 11.1 & 0.238 \\
\hline Response to name & 62 & 43.5 & 17 & 11.8 & $0.022^{*}$ \\
\hline Social smile & 33 & 51.5 & 6 & 16.7 & 0.190 \\
\hline Amount of social overtures (examiner) & 83 & 72.3 & 32 & 37.5 & $0.001 *$ \\
\hline Amount of social overtures (caregiver) & 48 & 55.1 & 16 & 6.3 & $0.001^{*}$ \\
\hline Amount of reciprocal communication & 42 & 66.7 & 23 & 30.4 & $0.009^{*}$ \\
\hline Functional play & 62 & 69.4 & 17 & 29.4 & $0.005^{*}$ \\
\hline Imaginative/creative play & 83 & 94.0 & 31 & 67.7 & $0.001^{*}$ \\
\hline Self-injury & 83 & 12.0 & 32 & 0.0 & 0.060 \\
\hline Anxiety & 83 & 36.1 & 31 & 35.5 & 0.999 \\
\hline
\end{tabular}

All $p$ values computed using Fisher's exact test

${ }^{*} p$ value remained $<0.05$ after adjusting for multiple testing using FDR

females with FM-only and PM/FM mosaicism did not differ significantly on any of the ADOS-2 CSS (Table 5).

Females with FM-only had significantly higher scores on the irritability, lethargy, hyperactivity and inappropriate speech subscales of the $\mathrm{ABC}-\mathrm{C}$, in addition to significantly higher overall scores and a significantly lower UI (Table 5), after adjusting for cFSIQ. All seven (100.0\%) females with PM/FM mosaicism fell in the very high or high UI category compared to twelve (44.4\%) females with FM-only; this difference was significant (Fisher's exact test, $p=0.046$ ). Of note, the female cohorts could not be stratified by age given the small number of females in the 13 and over age group.

\section{Discussion}

This study contributes to the field by providing a neurodevelopmental characterisation (ID, ASD and maladaptive behaviours) in individuals with FXS, stratified by sex and presence/absence of mosaicism. To the best of the authors' knowledge, this is the first study to compare these features between females with FM-only and with PM/FM mosaicism. Using a large international cohort of individuals with FXS, females were shown to have a milder clinical phenotype in comparison with males, with this attenuation in phenotype being more pronounced in females with PM/FM mosaicism. Similarly, males with PM/FM mosaicism also presented with a milder phenotype in comparison with their FM-only counterparts. The differences observed between sexes and allelic classes are primarily a result of the more severe cognitive impairment in males and FM-only individuals, respectively. These results are likely underpinned by FMR1-specific molecular differences between sex and allelic classes. Specifically, FMR1 promoter methylation mosaicism (reviewed in 
Table 4 Comparison of males by allelic class on corrected intellectual functioning, ASD features and maladaptive behaviours

\begin{tabular}{|c|c|c|c|c|c|}
\hline & \multicolumn{2}{|c|}{ FM-only } & \multicolumn{2}{|c|}{ PM/FM Mosaic } & \multirow[t]{2}{*}{$p$} \\
\hline & $n$ & Mean \pm SD & $n$ & Mean \pm SD & \\
\hline \multicolumn{6}{|l|}{ Intellectual functioning $^{1}$} \\
\hline $\mathrm{cVIQ}$ & 69 & $41.5 \pm 23.2$ & 18 & $59.6 \pm 19.7$ & 0.032 \\
\hline CPIQ & 69 & $42.1 \pm 20.3$ & 19 & $54.1 \pm 14.1$ & 0.138 \\
\hline cWMI & 25 & $30.0 \pm 11.4$ & 9 & $51.7 \pm 13.0$ & 0.075 \\
\hline CPSI & 34 & $38.9 \pm 11.6$ & 12 & $62.8 \pm 20.0$ & $0.004^{*}$ \\
\hline CFSIQ & 69 & $28.9 \pm 23.8$ & 18 & $49.9 \pm 18.1$ & $0.019^{*}$ \\
\hline \multicolumn{6}{|l|}{ ASD features ${ }^{2}$} \\
\hline ADOS CSS & 64 & $6.67 \pm 2.06$ & 16 & $6.00 \pm 2.31$ & 0.930 \\
\hline SA CSS & 64 & $6.38 \pm 2.26$ & 16 & $5.69 \pm 2.18$ & 0.658 \\
\hline RRB CSS & 64 & $7.64 \pm 1.92$ & 16 & $7.31 \pm 1.78$ & 0.710 \\
\hline \multicolumn{6}{|l|}{ Maladaptive behaviours ${ }^{3}$} \\
\hline Irritability & 70 & $15.5 \pm 11.4$ & 20 & $9.00 \pm 7.58$ & $0.011^{*}$ \\
\hline Lethargy & 70 & $6.71 \pm 5.42$ & 20 & $4.65 \pm 3.63$ & 0.117 \\
\hline Stereotypy & 70 & $5.60 \pm 4.66$ & 20 & $3.90 \pm 4.39$ & 0.233 \\
\hline Hyperactivity & 70 & $12.1 \pm 8.28$ & 20 & $8.35 \pm 7.63$ & 0.071 \\
\hline Inappropriate speech & 70 & $4.90 \pm 3.50$ & 20 & $2.80 \pm 3.27$ & $0.009^{*}$ \\
\hline Social avoidance & 70 & $3.47 \pm 2.75$ & 20 & $2.05 \pm 3.07$ & $0.012^{*}$ \\
\hline$A B C$ total & 70 & $48.3 \pm 28.3$ & 20 & $30.8 \pm 23.4$ & $0.012^{*}$ \\
\hline$A B C U I$ & 70 & $0.62 \pm 0.19$ & 20 & $0.73 \pm 0.16$ & $0.006^{*}$ \\
\hline
\end{tabular}

${ }^{1}$ Semi-parametric regression adjusted for country, age and ADOS CSS

${ }^{2}$ Robust regression adjusted for CFSIQ

${ }^{3}$ Robust regression adjusted for age

${ }^{*} p$ value remained $<0.05$ after adjustment for multiple testing using FDR

Kraan et al. [68]) has been inversely correlated with FMRP expression in blood and IQ scores in individuals with FXS of both sexes [60, 69, 70].

\section{Intellectual functioning}

Consistent with previous literature [13, 71], approximately $90 \%$ of males had FSIQ $<70$ compared with $47 \%$ of females. Males also had significantly lower corrected IQ scores on all domains compared with females, which remained significant when stratified by age. Significant variability was observed within both the male and female cohorts as reflected in the range of standard IQ scores (Additional file 1: Table S4).

Although males with FM-only and PM/FM mosaicism did not differ on the proportion having FSIQ $<70$, males with PM/FM mosaicism presented with higher corrected IQ scores on all domains though only cPSI and cFSIQ reached statistical significance. When using standard IQ scores VIQ, WMI and PSI were all significantly higher in the PM/FM mosaic group. Closer inspection of the data stratified by age revealed these significant differences were also observed in the younger age group $(<$ 13 years), but not in the older age group ( $\geq 13$ years),
Table 5 Comparison of females by allelic class on corrected intellectual functioning, ASD features and maladaptive behaviours

\begin{tabular}{|c|c|c|c|c|c|}
\hline & \multicolumn{2}{|c|}{ FM-only } & \multicolumn{2}{|c|}{ PM/FM Mosaic } & \multirow[b]{2}{*}{$p$} \\
\hline & $n$ & Mean \pm SD & $\bar{n}$ & Mean \pm SD & \\
\hline \multicolumn{6}{|l|}{ Intellectual functioning $^{1}$} \\
\hline $\mathrm{cVIQ}$ & 28 & $70.7 \pm 17.0$ & 8 & $85.9 \pm 20.0$ & $0.024^{*}$ \\
\hline CPIQ & 28 & $66.2 \pm 17.2$ & 8 & $78.8 \pm 12.3$ & $0.006^{*}$ \\
\hline cWMI & 9 & $59.8 \pm 17.2$ & 4 & $80.0 \pm 15.0$ & $0.021^{*}$ \\
\hline CPSI & 16 & $75.3 \pm 19.0$ & 6 & $92.7 \pm 18.1$ & $0.014^{*}$ \\
\hline CFSIQ & 28 & $64.5 \pm 17.3$ & 8 & $79.4 \pm 15.2$ & $0.019^{*}$ \\
\hline \multicolumn{6}{|l|}{ ASD features $^{2}$} \\
\hline ADOS CSS & 24 & $4.75 \pm 2.09$ & 8 & $3.88 \pm 2.85$ & 0.806 \\
\hline SA CSS & 24 & $4.71 \pm 2.03$ & 8 & $4.13 \pm 2.59$ & 0.664 \\
\hline RRB CSS & 24 & $6.67 \pm 2.30$ & 8 & $4.63 \pm 3.16$ & 0.523 \\
\hline \multicolumn{6}{|l|}{ Maladaptive behaviours ${ }^{2}$} \\
\hline Irritability & 27 & $11.8 \pm 12.0$ & 7 & $3.57 \pm 3.51$ & $0.036^{*}$ \\
\hline Lethargy & 27 & $5.44 \pm 5.73$ & 7 & $1.43 \pm 1.90$ & $0.037^{*}$ \\
\hline Stereotypy & 27 & $3.48 \pm 4.58$ & 7 & $0.00 \pm 0.00$ & NA \\
\hline Hyperactivity & 27 & $8.33 \pm 7.69$ & 7 & $0.86 \pm 0.90$ & $<0.001^{*}$ \\
\hline Inappropriate speech & 27 & $3.19 \pm 3.04$ & 7 & $0.29 \pm 0.49$ & $0.001^{*}$ \\
\hline Social avoidance & 27 & $2.67 \pm 3.34$ & 7 & $1.29 \pm 2.56$ & 0.429 \\
\hline ABC Total & 27 & $34.9 \pm 30.3$ & 7 & $7.43 \pm 6.40$ & $0.001^{*}$ \\
\hline$A B C U I$ & 27 & $0.69 \pm 0.21$ & 7 & $0.88 \pm 0.06$ & $0.005^{*}$ \\
\hline
\end{tabular}

${ }^{1}$ Robust regression adjusted for ADOS CSS

${ }^{2}$ Robust regression adjusted for CFSIQ

${ }^{*} p$ value remained $<0.05$ after adjustment for multiple testing using FDR

likely due to the small sample size of PM/FM mosaic males $(n=6)$ in the older age bracket. Thus, while the majority of males still had IQ scores in the extremely low range, males with PM/FM mosaicism tended to have IQ scores within the milder ID range. The subtle differences that were observed between these two groups (FM-only versus PM/FM mosaic) when comparing index scores demonstrate the significant impact the floor effect has on exploring the phenotype in males with FXS, though there was less impact in females with FXS. Females with PM/FM mosaicism were less likely to have FSIQ $<70$ compared with females with FM-only, and all corrected IQ scores were significantly higher in the PM/ FM mosaic group.

\section{ASD features}

Males were more likely to meet criteria for ASD on the ADOS-2 compared with females, though within each sex, the proportion of FM-only and PM/FM mosaic cases meeting ADOS-2 criteria did not significantly differ. When using gold standard diagnostic instruments (i.e. ADOS, ADI-R and DSM criteria) as many as $60 \%$ of males with FXS presented with behaviours that supported a comorbid diagnosis of ASD [72]. Thus, the proportion 
of those meeting criteria for ASD in the current study (males $90.4 \%$ and females 65.6\%) are somewhat higher than previous estimates, though are similar to reports on the prevalence of ASD symptoms more generally in children with FXS (approximately $90 \%$ of males and a third of females) [11, 25]. Nonetheless, the proportion meeting ADOS-2 criteria for ASD is not an accurate reflection of the proportion of individuals with FXS who would meet DSM-5 criteria for ASD.

Significant discordance has been observed even between gold standard diagnostic assessments when determining the presence of ASD in those with FXS. In particular, one study demonstrated good agreement between ADOS-G and DSM-IV criteria [72], while another found a high false positive rate using the ADOS-G compared with ADI-R and DSM-IV criteria combined [20]. The revised diagnostic algorithms of the ADOS-2 and changes to ASD diagnostic criteria within the DSM-5 complicate this picture further. The diagnostic algorithm of the ADOS-G did not incorporate repetitive and restricted behaviours and given that these types of behaviours, particularly hand flapping and perseveration of speech, are common in those with FXS [73], the ADOS2 may increase rates of ASD in FXS in comparison with ADOS-G. Moreover, a recent longitudinal study of ASD in FXS demonstrated increased rates of ASD over time, with $80 \%$ of males $\left(M_{\text {age }}=11.50\right.$ years $)$ and $41.7 \%$ of females $\left(M_{\text {age }}=11.21\right.$ years $)$ meeting criteria for ASD at time 2 using the ADOS-2 algorithms [24]. Similarly, a non-significant trend for social affect CSS scores to increase with chronological age was observed in another longitudinal study of males with FXS [27]. Thus, the use of the ADOS-2 in addition to the wide age range of participants in the current study may explain the elevated rates and features associated with ASD.

Thurman and colleagues [27] also demonstrated that increases in nonverbal ability were associated with decreases in SA CSS and RRB CSS in their longitudinal study of males with FXS (FM-only + PM/FM mosaic). We also found significant associations between nonverbal IQ (CPIQ) and both SA and RBB CSS in males with FXS, and between cPIQ and RRB CSS in females. It has been suggested that individuals with better nonverbal abilities may have a greater range of interests and activities, and may also be more able to engage in more productive rather than repetitive behaviours compared with children with poorer nonverbal abilities [27, 74]. Expressive language skills were also associated with less repetitive behaviours in males with FXS [27]. However, in the current study, associations with cVIQ and RRB CSS were only found in the female cohort. The very low verbal ability scores obtained using the Wechsler scales to assess VIQ may explain the lack of associations in our male cohort.
No differences were observed between sexes on ADOS-2 CSS after controlling for cFSIQ. Thus, the increased prevalence of features associated with ASD in males with FXS compared with females is likely due to the greater severity of intellectual impairment. In line with this, when we adjusted for ADOS CSS for comparisons of intellectual functioning, the differences between males and females remained significant. Nonetheless, differences were obtained at an item level between males and females, particularly the social interaction items. Females were more likely to effectively use eye contact, initiate joint attention, respond to their name, share enjoyment and show objects and toys of interest to the examiner/caregiver. Moreover, females more frequently attempted to engage the examiner (e.g. amount of social overtures) and their caregivers and displayed more creative play skills in comparison with males. More specifically, only a very small proportion of females (6.3\%) showed atypical amount of social overtures to the caregiver and for most females, initiation of joint attention and amount of reciprocal communication was typical. Yet, approximately $75 \%$ of females presented with atypical quality of social overtures. This is consistent with the view that individuals with FXS are interested in engaging socially with others, though other factors such as anxiety and hyperarousal may interfere with the quality of these social interactions [75]. As described by Mazzocco and colleagues [76], language abilities can have negative impacts on social interactions, even in brief encounters with an unfamiliar adult. Specifically, girls with FXS $(n=20$; VIQ $>70)$ asked fewer questions overall, fewer questions that logically flowed from the previous utterance, and spoke more repetitious phrases compared with typically developing peers; factors that would contribute to poorer quality of social overtures in an ADOS-2 assessment. Turkstra and colleagues [77] also demonstrated that the gap in performance on social cognition tasks for females with FXS compared with typically developing peers is likely attributed to general cognitive functions such as language and IQ. This phenomenon is likely further inflated in males with FXS, given their more impaired verbal abilities. Interventions that target the contributions of cognitive and language deficits may assist with social interaction skills.

Interestingly, the proportion of males and females presenting with repetitive and restricted behaviours only differed at the item level for unusual sensory interests, with more males engaging in such behaviours compared with females. The lack of differences on repetitive behaviours, as well as hand mannerisms is not surprising given that these are typical features that are associated with FXS in both males [78] and females [17, 18]. In particular, preoccupations with one particular or parts of object(s), distress due to environmental changes and a 
restricted range of interests have been reported for both males and females with FXS [17]. These item-level differences remained significantly different between males and females for the younger age group ( $<13$ years), but not the older age group ( $\geq 13$ years); this is likely due to the small sample of females in the older age group. Increased rates of RRBs can further impede daily functioning by creating a barrier to learning and social interaction [79]. Thus, interventions that aim to alleviate these behaviours would also be beneficial. However, identification of the mechanism for the specific RRB would need to be targeted (e.g. cognitive ability, anxiety), and this likely varies for each individual [78].

These findings are in contrast to a study comparing males and females with idiopathic ASD [33], where females who met criteria for ASD on a 'gold standard' measure (i.e. ADOS-2 or ADI-R) had similar profiles to males on the ADOS-2 after adjusting for multiple comparisons. However, females were reported by their parents to have greater autistic traits compared to males on the Social Responsiveness Scale (SRS) [80]. This may be due to the fact that the SRS provides norms for males and females separately, while the ADOS-2 is normed predominantly on a sample of males with idiopathic ASD. To further elucidate the autistic profile in females with FXS, the use of other assessment tools such as the SRS may be beneficial.

No differences were observed between FM-only and PM/FM mosaic cases on any ADOS CSS in either sex, after adjusting for cFSIQ. Other researchers investigating the associations between FXS-specific molecular variables and ASD phenomenology reported that FMRP did not account for unique variance in predicting ASD symptom severity over and above the contribution of nonverbal IQ [81]. However, in our recent study on a sub-sample of those individuals included in the current study, we demonstrated that those FM-only males who had incomplete silencing of FMR1 mRNA FM alleles had greater autistic features based on the ADOS CSS [39]. This suggested that overexpression of expanded FM mRNA may contribute towards the ASD features seen in this sub-group of males with FXS. However, these findings need replication in larger independent cohorts.

\section{Maladaptive behaviours}

Although maladaptive behaviours did not significantly differ between males and females after adjusting for cFSIQ, significant differences were observed between FM-only and PM/FM mosaic cases within each of the sexes. In particular, males with FM-only were reported by their caregivers to be more irritable, use more inappropriate speech and to be more socially avoidant compared with males with PM/FM mosaicism.
Moreover, the quality of life was reported to be significantly poorer in FM-only males. When stratified by age, no significant differences were observed between these two groups for the under 13 age group. While significant differences were observed for the older age group on all the $\mathrm{ABC}-\mathrm{C}$ subscales, except hyperactivity. However, these findings should be interpreted with caution given only six males with PM/FM mosaicism wereincluded in the analysis.

Similarly, females with FM-only were reported to be more irritable, lethargic and hyperactive and to use more inappropriate speech compared with their PM/FM mosaic counterparts. However, the two female groups did not differ on the social avoidance subscale which is consistent with the more social approach type behaviours that were observed in the ADOS-2. Of note, all females with PM/FM mosaicism were reported to have high or very high quality of life compared with only $44 \%$ of those in the female FM-only group. Thus, parent reports of behaviours appear to significantly differ between allelic classes despite no significant differences being observed on the ADOS-2. Unlike the ADOS-2 administrators/ coders, parents are not blinded to allelic classification and consequently may be biased in their responses on the $\mathrm{ABC}-\mathrm{C}$ given the prognostic information they are given at the time of diagnosis.

\section{Limitations}

An important limitation of the current study is the use of several assessment types for intellectual functioning. Specifically, the WISC-III Chilean edition is not directly comparable with the other Wechsler scales used in this study. Further, while the MSEL has demonstrated good convergent validity with other intellectual functioning measures in a sample of children with ASD [59], the MSEL may overestimate cognitive abilities in comparison with the Wechsler scales, particularly in children with FXS. Behavioural challenges that emerge later in childhood including inattention and hyperactivity may impact the child's ability to complete more structured intellectual functioning assessments such as the Wechsler scales.

Evidence for the influence of these assessments is demonstrated when significant differences between allelic classifications within the male cohort were lost when the MSEL and WISC-III were removed. Within the WISC-III Coding and Arithmetic are included in the performance and verbal indexes, respectively. Coding assesses processing speed skills while Arithmeticassesses working memory. Working memory and processing speed tasks can be particularly difficult for males with FXS. The significant flooring associated with males with FXS has a clear impact on scores and thus the understanding of the variability in the phenotype. Thus, there 
is still a great need to develop an appropriate and accurate assessment battery that can be used longitudinally and that is inclusive for all cognitive abilities. Such an assessment battery will assist in understanding developmental trajectories of individuals with FXS but will also be extremely beneficial for clinical trials. While there is good convergent validity between each of Wechsler scales according to the test manuals [54-56], this might not hold for individuals with ID. Thus, the development and use of a single intellectual functioning assessment tool that is sensitive to variation of cognitive skills in individuals of a wide range, from infancy to adulthood, will be beneficial for future research and clinical practice.

Another limitation to the current study is the wide age range of the participants (1-43 years). While age was used as a covariate (where appropriate) and analyses were also conducted with the groups stratified into two age groups, the small number of females in the older age group prevented further stratification into three categories: (i) childhood ( $<13$ years); (ii) adolescence ( $\geq 13$ years and $\leq$ 18 years); (iii) adulthood (19+ years). Future research with larger samples of females would be beneficial. Moreover, detection of ASD features may be more difficult to detect in those aged under 2 years. Nonetheless, for those children in the current study, four children were aged under 2 years and completed a Toddler module of the ADOS-2. All these children met the requirements of this module, cruising/ walking and based on the MSEL, a nonverbal mental age greater than 12 months [82]. Two of these children fell in the mild-to-moderate range of concern, one in the moderate-to-severe range of concern and one in the littleto-no concern range. Suggesting that ASD features were accurately detected for participants $<2$ years of age.

Lastly, this is not a population-based study and recruitment was consequently confounded by site of recruitment. There is a likely ascertainment bias, particularly in the female group, in which more severe females were involved in the study. This may contribute towards the significant differences that were observed between FM-only and PM/FM mosaic cases. This is an important issue for the broader FXS field and is not unique to this study. Further, obtaining large samples of individuals with PM/FM mosaicism is difficult, as evidenced by this study and other previous studies including males with mosaicism. The small sample of individuals with PM/FM mosaicism in the current study likely reduced statistical power, particularly when groups were stratified by age. Further studies in unbiased and larger samples of females with FM-only and PM/FM mosaicism are needed.

\section{Conclusions}

This study contributes to the field by providing a neurodevelopmental characterisation (ID, ASD and maladaptive behaviours) in individuals with FXS stratified by sex and presence/absence of mosaicism. To the best of the authors' knowledge, there is no study to date that has compared females with FM-only and females with PM/FM mosaicism on multiple psychological domains using standardised measures. Moreover, there is a lack of research that has compared specific behaviours associated with ASD between males and females. Of note, the findings regarding the differences between males and females on maladaptive behaviours were lost after controlling for IQ, which has not previously been explored.

This study demonstrates that while males with FXS had significantly lower intellectual functioning scores than females with FXS, no significant differences were observed on ASD features after controlling for cFSIQ. This finding suggests that it is primarily the significant cognitive impairment in males with FXS that lead to the increased prevalence of ASD features in comparison with females. Overall ASD features were also shown to be significantly and negatively correlated with cFSIQ scores, in each sex. Taken together, these findings are consistent with the theory that the degree of cognitive impairments in genetic conditions account for the ASD phenomenology via reduced intellectual abilities [83], possibly due to common biochemical and neural dysfunctions leading to both these phenotypic characteristics. These findings have implications in genetic counselling and clinical settings. Nonetheless, the contribution of masking, particularly in higher functioning females with FXS, should be considered and assessments with other ASD assessment tools, which provide norms for each sex may assist to elucidate symptomatology in females.

The $\mathrm{ABC}-\mathrm{C}$ is widely used as a primary outcome measure in clinical trials in FXS. Given the significant differences seen between the ADOS- 2 and ABC-C between allelic classes, caution is advised for the $\mathrm{ABC}-\mathrm{C}$ as a primary outcome measure when used in isolation. Moreover, current behavioural interventions and treatments for FXS are primarily those that are recommended for individuals with idiopathic ASD and consequently, there is a clear unmet need for treatments that are specific to FXS. Based on the findings of the current study, interventions that target cognitive abilities, particularly nonverbal skills, may in turn reduce the severity of ASD features and other maladaptive behaviours. Reductions in these behaviours have the potential to lead to better outcomes for the affected children and their families.

\section{Supplementary information}

Supplementary information accompanies this paper at https://doi.org/10. 1186/s11689-019-9288-7.

Additional file 1. Note S1-S4 and Table S1-S15 Clinical Phenotype of FXS. 


\section{Abbreviations}

ABC-C: Aberrant Behavior Checklist-Community; ADI-R: Autism Diagnostic Interview-Revised; ADOS-G: Autism Diagnostic Observation Schedule-Generic; ADOS-2: Autism Diagnostic Observation Schedule-2nd Edition; ADOS CSS: Overall ADOS-2 Calibrated Severity Score; ASD: Autism spectrum disorder; CFSIQ: Corrected Full Scale IQ; CPIQ: Corrected performance IQ; CPSI: Corrected processing speed index; CSS: Calibrated severity score; CVIQ: Corrected verbal IQ; CWMI: Corrected working memory index; DSM5: Diagnostic and Statistical Manual of Mental Health Disorders-5th Edition; DSM-IV: Diagnostic and Statistical Manual of Mental Health Disorders-4th Edition; FDR: False discovery rate; FM: Full mutation; FMRP: Fragile X mental retardation protein; FSIQ: Full Scale IQ; FXS: Fragile X syndrome; MSEL: Mullen Scales of Early Learning; PIQ: Performance IQ; PM: Pre-mutation; PM/FM: Premutation/full mutation mosaic; PSI: Processing speed index; RRB CSS: Repetitive and Restricted Behaviour Calibrated Severity Scores; SA CSS: Social Affect Calibrated Severity Scores; VIQ: Verbal IQ; WAIS-IV: Wechsler Adult Intelligence Scale-Fourth Edition; WISC-III: Wechsler Intelligence Scale for Children-Third Edition; WISC-IV: Wechsler Intelligence Scale for ChildrenFourth Edition; WMI: Working memory index; WPPSI-III: Wechsler Preschool and Primary Scale of Intelligence-Third Edition

\section{Acknowledgements}

The authors would like to thank all the study participants and their families for being involved in the study. We would also like to thank the following individuals for their assistance with the administration and coding of ADOS assessments: Cherie Green, Nusrat Ahmed, Annabelle May Marsh, Jaqueline Maya and Pura Ballester Navarro.

\section{Authors' contributions}

$E B, M A, S M A, C M K, L B, H S, D J A$ and DEG all contributed to the conception and design of the study, data acquisition, data analysis and interpretation of data. $\mathrm{MB}$ and $\mathrm{EB}$ conducted data analysis and interpretation of the data. $\mathrm{LB}$, $A U$ and DEG provided supervision of assessments. DF, MH, JE, CR, MJF, JC, $L M, V F, B C, P M, C T$, IS and $A A$ all contributed to patient recruitment/ acquisition of data. All authors have been involved in the drafting of the manuscript and/or revising it critically for important intellectual content and have read and approved the final manuscript.

\section{Funding}

This study was supported by the Victorian Government's Operational Infrastructure Support Program, with the salaries supported by NHMRC project grants (no. 1049299 and no. 1103389 to DEG; and no. 1120561 to CMK); Murdoch Children's Research Institute, Royal Children's Hospital Foundation (DEG); Next Generation Clinical Researchers Program - Career Development Fellowship, funded by the Medical Research Future Fund (MRF1141334 to D.E.G.) and the Financial Markets Foundation for Children (Australia) (no. 2017 - 361 to DEG, CMK and DJA); MJF and CR were supported by the Genetics of Learning Disability (GOLD) Service. MA was supported by the International Postgraduate Research Scholarships (IPRS) and the Research Training Program Fee offset scholarship funded by the Australian Government and awarded by the University of Melbourne, and in part by the Diagnosis and Development group of the Murdoch Children's Research Institute. SMA was funded by the CONICYT and Chile's National Commission for Scientific and Technological Research.

\section{Availability of data and materials}

The datasets used and/or analysed during the current study are available from the corresponding author on reasonable request.

\section{Ethics approval and consent to participate}

All procedures were approved by The Royal Children's Hospital and INTA Human Research Ethics Committees (HREC \#33066 and \#15, respectively). All parents/caregivers provided written informed consent and those who were deemed cognitively able, also provided written informed consent.

\section{Consent for publication}

Not applicable

\section{Competing interests}

David Eugeny Godler is named as an inventor on patent applications [PCT/ AU2010/000169, PCT/AU2011/001024 and PCT/AU2014/000044] related to the technology developed to diagnose fragile $X$ syndrome. All other authors declare no other competing interests.

\section{Author details}

'Diagnosis and Development, Murdoch Children's Research Institute, Royal Children's Hospital, Melbourne, VIC, Australia. ${ }^{2}$ Faculty of Medicine, Dentistry and Health Sciences, Department of Paediatrics, University of Melbourne, Parkville, VIC, Australia. ${ }^{3}$ School of Psychology and Public Health, La Trobe University, Bundoora, VIC, Australia. 'Brain and Mind, Murdoch Children's Research Institute, Royal Children's Hospital, Melbourne, VIC, Australia. ${ }^{5}$ Neurodisability and Rehabilitation, Murdoch Children's Research Institute, Royal Children's Hospital, Melbourne, VIC, Australia. ${ }^{6}$ Royal Children's Hospital, Melbourne, VIC, Australia. 'Department of Pediatrics, Monash University, Clayton, VIC, Australia. ${ }^{8}$ Centre for Epidemiology and Biostatistics, Melbourne School of Population and Global Health, University of Melbourne, Carlton, VIC, Australia. 'Victorian Clinical Genetics Services and Murdoch Children's Research Institute, Royal Children's Hospital, Melbourne, VIC, Australia.

${ }^{10}$ Monash Genetics, Monash Health, Melbourne, VIC, Australia. ${ }^{11}$ Genetics of Learning Disability Service, Hunter Genetics, Waratah, NSW, Australia.

${ }^{12}$ Fragile X Alliance Inc, Centre for Developmental Disability Health Victoria, Monash University, North Caulfield, Clayton, VIC, Australia. ${ }^{13}$ Laboratory of Molecular Cytogenetics, Department of Genetics and Metabolic Diseases, Institute of Nutrition and Food Technology (INTA), University of Chile, Santiago, Chile.

Received: 30 January 2019 Accepted: 7 November 2019 Published online: 26 December 2019

\section{References}

1. Monaghan KG, Lyon E, Spector EB. erican College of Medical G, Genomics ACMG standards and guidelines for fragile $X$ testing: a revision to the disease-specific supplements to the Standards and Guidelines for Clinical Genetics Laboratories of the American College of Medical Genetics and Genomics. Genet Med. 2013;15(7):575-86.

2. Verkerk AJ, Pieretti $M$, Sutcliffe JS, Fu YH, Kuhl DP, Pizzuti A, et al. Identification of a gene (FMR-1) containing a CGG repeat coincident with a breakpoint cluster region exhibiting length variation in fragile $X$ syndrome. Cell. 1991;65(5):905-14.

3. Pieretti M, Zhang FP, Fu YH, Warren ST, Oostra BA, Caskey CT, et al. Absence of expression of the FMR-1 gene in fragile X syndrome. Cell. 1991;66(4):817-22.

4. Parikshak NN, Luo R, Zhang A, Won H, Lowe JK, Chandran V, et al. Integrative functional genomic analyses implicate specific molecular pathways and circuits in autism. Cell. 2013;155(5):1008-21.

5. Bassell GJ, Warren ST. Fragile $X$ syndrome: loss of local mRNA regulation alters synaptic development and function. Neuron. 2008;60(2):201-14.

6. Nolin SL, Glicksman A, Houck GE Jr, Brown WT, Dobkin CS. Mosaicism in fragile X affected males. Am J Med Genet. 1994;51(4):509-12.

7. Rousseau F, Heitz D, Biancalana V, Blumenfeld S, Kretz C, Boue J, et al. Direct diagnosis by DNA analysis of the fragile $X$ syndrome of mental retardation. N Engl J Med. 1991;325(24):1673-81.

8. Rousseau F, Heitz D, Tarleton J, MacPherson J, Malmgren H, Dahl N, et al. A multicenter study on genotype-phenotype correlations in the fragile $X$ syndrome, using direct diagnosis with probe StB12.3: the first 2,253 cases. Am J Hum Genet. 1994;55(2):225-37.

9. Godler DE, Inaba Y, Shi EZ, Skinner C, Bui QM, Francis D, et al. Relationships between age and epi-genotype of the FMR1 exon 1/intron 1 boundary are consistent with non-random X-chromosome inactivation in FM individuals, with the selection for the unmethylated state being most significant between birth and puberty. Hum Mol Genet. 2013:22(8):1516-24.

10. Backes M, Genc B, Schreck J, Doerfler W, Lehmkuhl G, von Gontard A. Cognitive and behavioral profile of fragile $X$ boys: correlations to molecular data. Am J Med Genet. 2000;95(2):150-6.

11. Bailey DB Jr, Hatton DD, Skinner M, Mesibov G. Autistic behavior, FMR1 protein, and developmental trajectories in young males with fragile $X$ syndrome. J Autism Dev Disord. 2001;31(2):165-74.

12. Bartholomay $\mathrm{KL}$, Lee $\mathrm{CH}$, Bruno JL, Lightbody AA, Reiss AL. Closing the gender gap in fragile $X$ syndrome: review of females with fragile $X$ syndrome and preliminary research findings. Brain Sciences. 2019;9(1):11.

13. de Vries BB, Wiegers AM, Smits AP, Mohkamsing S, Duivenvoorden HJ, Fryns JP, et al. Mental status of females with an FMR1 gene full mutation. Am J Hum Genet. 1996;58(5):1025-32. 
14. Quintin EM, Jo B, Hall SS, Bruno JL, Chromik LC, Raman MM, et al. The cognitive developmental profile associated with fragile $X$ syndrome: a longitudinal investigation of cognitive strengths and weaknesses through childhood and adolescence. Dev Psychopathol. 2016;28(4 pt2):1457-69.

15. Hartley SL, Seltzer MM, Raspa M, Olmstead M, Bishop E, Bailey DB. Exploring the adult life of men and women with fragile $X$ syndrome: results from a national survey. Am J Intellect Dev Disabil. 2011;116(1):16-35.

16. Keysor CS, Mazzocco MMM, McLeod DR, Hoehn-Saric R. Physiological arousal in females with fragile $X$ or Turner syndrome. Dev Psychobiol. 2002;41(2):133-46.

17. Mazzocco MM, Kates WR, Baumgardner TL, Freund LS, Reiss AL. Autistic behaviors among girls with fragile $X$ syndrome. J Autism Dev Disord. 1997:27(4):415-35

18. Freund LS, Reiss AL, Abrams MT. Psychiatric disorders associated with fragile $X$ in the young female. Pediatrics. 1993;91(2):321-9.

19. Ciaccio C, Fontana L, Milani D, Tabano S, Miozzo M, Esposito S. Fragile X syndrome: a review of clinical and molecular diagnoses. Ital J Pediatr. 2017;43:39.

20. Hernandez RN, Feinberg RL, Vaurio R, Passanante NM, Thompson RE, Kaufmann WE. Autism spectrum disorder in fragile $X$ syndrome: a longitudinal evaluation. Am J Med Genet A. 2009;149A(6):1125-37.

21. Cohen IL, Nolin SL, Sudhalter V, Ding XH, Dobkin CS, Brown WT. Mosaicism for the FMR1 gene influences adaptive skills development in fragile $X$ affected males. Am J Med Genet. 1996;64(2):365-9.

22. Aman MG, Singh NN, Stewart AW, Field CJ. The aberrant behavior checklist: a behavior rating scale for the assessment of treatment effects. Am J Ment Defic. 1985;89(5):485-91.

23. Hustyi KM, Hall SS, Jo B, Lightbody AA, Reiss AL. Longitudinal trajectories of aberrant behavior in fragile X syndrome. Res Dev Disabil. 2014;35(11):2691-701.

24. Lee M, Martin GE, Berry-Kravis E, Losh M. A developmental, longitudinal investigation of autism phenotypic profiles in fragile $X$ syndrome. J Neurodev Disord. 2016;8(1):47.

25. Hatton DD, Sideris J, Skinner M, Mankowski J, Bailey DB Jr, Roberts J, et al. Autistic behavior in children with fragile $X$ syndrome: prevalence, stability, and the impact of FMRP. Am J Med Genet A. 2006;140a(17):1804-13.

26. Hall SS, Lightbody AA, Hirt M, Rezvani A, Reiss AL. Autism in fragile $X$ syndrome: a category mistake? J Am Acad Child Adolesc Psychiatry. 2010; 49(9):921-33.

27. Thurman AJ, McDuffie A, Kover ST, Hagerman RJ, Abbeduto L. Autism symptomatology in boys with fragile $X$ syndrome: a cross sectional developmental trajectories comparison with nonsyndromic autism spectrum disorder. J Autism Dev Disord. 2015;45(9):2816-32.

28. Richards C, Jones C, Groves L, Moss J, Oliver C. Prevalence of autism spectrum disorder phenomenology in genetic disorders: a systematic review and meta-analysis. Lancet Psychiatry. 2015;2(10):909-16.

29. Kidd SA, Lachiewicz A, Barbouth D, Blitz RK, Delahunty C, McBrien D, et al. Fragile $X$ syndrome: a review of associated medical problems. Pediatrics. 2014;134:995.

30. Ferri SL, Abel T, Brodkin ES. Sex differences in autism spectrum disorder: a review. Curr Psychiatry Rep. 2018;20(2):9.

31. Christensen DL, Braun KVN, Baio J, Bilder D, Charles J, Constantino JN, et al. Prevalence and characteristics of autism spectrum disorder among children aged 8 years - Autism and Developmental Disabilities Monitoring Network, 11 Sites, United States, 2012. MMWR Surveill Summ (Washington, DC : 2002). 2018;65(13):1-23.

32. Loomes R, Hull L, Mandy WPL. What is the male-to-female ratio in autism spectrum disorder? A systematic review and meta-analysis. J Am Acad Child Adolesc Psychiatry. 2017:56(6):466-74

33. Ratto AB, Kenworthy L, Yerys BE, Bascom J, Wieckowski AT, White SW, et al. What about the girls? Sex-based differences in autistic traits and adaptive skills. J Autism Dev Disord. 2018;48(5):1698-711.

34. Kaufmann WE, Abrams MT, Chen W, Reiss AL. Genotype, molecular phenotype, and cognitive phenotype: correlations in fragile $X$ syndrome. Am J Med Genet. 1999;83(4):286-95

35. Tassone F, Hagerman RJ, Ikle DN, Dyer PN, Lampe M, Willemsen R, et al. FMRP expression as a potential prognostic indicator in fragile $X$ syndrome. Am J Med Genet. 1999:84(3):250-61

36. Hall S, DeBernardis M, Reiss AL. Social escape behaviors in children with fragile X syndrome. J Autism Dev Disord. 2006;36(7):935-47.

37. Loesch DZ, Bui QM, Dissanayake C, Clifford S, Gould E, Bulhak-Paterson D, et al. Molecular and cognitive predictors of the continuum of autistic behaviours in fragile X. Neurosci Biobehav Rev. 2007;31(3):315-26.
38. Lord C, Rutter M, Le Couteur A. Autism diagnostic interview-revised: a revised version of a diagnostic interview for caregivers of individuals with possible pervasive developmental disorders. J Autism Dev Disord. 1994; 24(5):659-85.

39. Baker EK, Arpone M, Aliaga SM, Bretherton L, Kraan CM, Bui M, et al Incomplete silencing of full mutation alleles in males with fragile $X$ syndrome is associated with autistic features. Mol Autism. 2019;10(1):21.

40. Loesch DZ, Huggins RM, Hagerman RJ. Phenotypic variation and FMRP levels in fragile X. Ment Retard Dev Disabil Res Rev. 2004;10(1):31-41.

41. Hessl D, Dyer-Friedman J, Glaser B, Wisbeck J, Barajas RG, Taylor A, et al. The influence of environmental and genetic factors on behavior problems and autistic symptoms in boys and girls with fragile $X$ syndrome. Pediatrics. 2001;108(5):E88.

42. Lessard M, Chouiali A, Drouin R, Sebire G, Corbin F. Quantitative measurement of FMRP in blood platelets as a new screening test for fragile X syndrome. Clin Genet. 2012;82(5):472-7.

43. Dyer-Friedman J, Glaser B, Hessl D, Johnston C, Huffman LC, Taylor A, et al. Genetic and environmental influences on the cognitive outcomes of children with fragile $X$ syndrome. J Am Acad Child Adolesc Psychiatry. 2002;41(3):237-44.

44. Godler DE, Slater HR, Bui QM, Storey E, Ono MY, Gehling F, et al. Fragile X mental retardation 1 (FMR1) intron 1 methylation in blood predicts verbal cognitive impairment in female carriers of expanded FMR1 alleles: evidence from a pilot study. Clin Chem. 2012;58(3):590-8.

45. Lightbody AA, Hall SS, Reiss AL. Chronological age, but not FMRP levels, predicts neuropsychological performance in girls with fragile $X$ syndrome. Am J Med Genet B Neuropsychiatr Genet. 2006;141b(5):468-72

46. Saluto A, Brussino A, Tassone F, Arduino C, Cagnoli C, Pappi P, et al. An enhanced polymerase chain reaction assay to detect pre- and full mutation alleles of the fragile X mental retardation 1 gene. J Mol Diagn. 2005;7(5):605-12.

47. Khaniani MS, Kalitsis P, Burgess T, Slater HR. An improved diagnostic PCR assay for identification of cryptic heterozygosity for CGG triplet repeat alleles in the fragile $X$ gene (FMR1). Mol Cytogenet. 2008;1:5.

48. Alliende MA, Urzua B, Valiente A, Cortes F, Curotto B, Rojas C. Direct molecular analysis of FMR-1 gene mutation in patients with fragile $\mathrm{Xq}$ syndrome and their families. Rev Med Chil. 1998;126(12):1435-46.

49. Aliaga SM, Slater HR, Francis D, Du Sart D, Li X, Amor DJ, et al. Identification of males with cryptic fragile $X$ alleles by methylation-specific quantitative melt analysis. Clin Chem. 2016;62(2):343-52.

50. Chen L, Hadd A, Sah S, Houghton JF, Filipovic-Sadic S, Zhang W, et al. Highresolution methylation polymerase chain reaction for fragile $X$ analysis: evidence for novel FMR1 methylation patterns undetected in Southern blot analyses. Genet Med. 2011;13(6):528-38.

51. Mullen EM. Mullen Scales of Early Learning. MN: American Guidance Service; 1995.

52. Wechsler D. Wechsler Preschool and Primary Scale of Intelligence - Third Edition Australian Standardised Edition. NSW: NCS Pearson Inc: 2004.

53. Wechsler D. Wechsler Preschool and Primary Scale of Intelligence-Third Edition Mexican Edition. Harcourt Assessment: USA; 2002

54. Wechsler D. Wechsler Intelligence Scale for Children-Fourth Edition Australian Standardised Edition. NSW: NCS Pearson Inc.; 2003.

55. Wechsler D. Wechsler Intelligence Scale for Children-Third Edition Chilean Edition. USA: The Psychological Corporation; 2007.

56. Wechsler D. Wechsler Adult Intelligence Scale_-Fourth Edition Australian and New Zealand Language Adaptation. NSW: NCS Pearson Inc.; 2008.

57. Wechsler D. Wechsler Adult Intelligence Scale-Fourth Edition Chilean Edition. NCS Pearson Inc: USA; 2008.

58. Richler J, Bishop SL, Kleinke JR, Lord C. Restricted and repetitive behaviors in young children with autism spectrum disorders. J Autism Dev Disord. 2007:37(1):73-85.

59. Bishop SL, Guthrie W, Coffing M, Lord C. Convergent validity of the Mullen Scales of Early Learning and the differential ability scales in children with autism spectrum disorders. Am J Intellect Dev Disabil. 2011;116(5):331-43.

60. Arpone M, Baker EK, Bretherton L, Bui M, Li X, Whitaker S, et al. Intragenic DNA methylation in buccal epithelial cells and intellectual functioning in a paediatric cohort of males with fragile X. Sci Rep. 2018;8(1):3644.

61. Whitaker S, Gordon S. Floor effects on the WISC-IV. Int J Dev Disabil. 2012 58(2):111-9

62. Lord C, Rutter M, DiLavore PC, Risi S, Gotham K, Bishop SL. Autism Diagnostic Observation Schedule, 2nd Edition (ADOS-2). Western Psychological Services: Los Angeles; 2012.

63. Hus V, Gotham K, Lord C. Standardizing ADOS domain scores: separating severity of social affect and restricted and repetitive behaviors. J Autism Dev Disord. 2014;44(10):2400-12. 
64. Hus V, Lord C. The Autism Diagnostic Observation Schedule, module 4: revised algorithm and standardized severity scores. J Autism Dev Disord. 2014;44(8):1996-2012.

65. Esler AN, Bal VH, Guthrie W, Wetherby A, Ellis Weismer S, Lord C. The Autism Diagnostic Observation Schedule, Toddler Module: Standardized Severity Scores. J Autism Dev Disord. 2015;45(9):2704-20.

66. Sansone SM, Widaman KF, Hall SS, Reiss AL, Lightbody A, Kaufmann WE, et al. Psychometric study of the Aberrant Behavior Checklist in fragile $X$ syndrome and implications for targeted treatment. J Autism Dev Disord. 2012;42(7):1377-92.

67. Kerr C, Breheny K, Lloyd A, Brazier J, Bailey DB Jr, Berry-Kravis E, et al. Developing a utility index for the Aberrant Behavior Checklist (ABC-C) for fragile X syndrome. Qual Life Res. 2015;24(2):305-14.

68. Kraan CM, Godler DE, Amor DJ. Epigenetics of fragile X syndrome and fragile X-related disorders. Dev Med Child Neurol. 2019:61(2):121-7.

69. Inaba Y, Schwartz CE, Bui QM, Li X, Skinner C, Field M, et al. Early detection of fragile $X$ syndrome: applications of a novel approach for improved quantitative methylation analysis in venous blood and newborn blood spots. Clin Chem. 2014;60(7):963-73.

70. Godler DE, Tassone F, Loesch DZ, Taylor AK, Gehling F, Hagerman RJ, et al. Methylation of novel markers of fragile $X$ alleles is inversely correlated with FMRP expression and FMR1 activation ratio. Hum Mol Genet. 2010;19(8): 1618-32.

71. Hessl D, Nguyen DV, Green C, Chavez A, Tassone F, Hagerman RJ, et al. A solution to limitations of cognitive testing in children with intellectual disabilities: the case of fragile X syndrome. J Neurodev Disord. 2009:1(1):33-45.

72. Harris SW, HessI D, Goodlin-Jones B, Ferranti J, Bacalman S, Barbato I, et al. Autism profiles of males with fragile X syndrome. Am J Ment Retard. 2008; 113(6):427-38

73. Hagerman RJ, Jackson AW 3rd, Levitas A, Rimland B, Braden M. An analysis of autism in fifty males with the fragile X syndrome. Am J Med Genet. 1986; 23(1-2):359-74.

74. Bishop SL, Richler J, Lord C. Association between restricted and repetitive behaviors and nonverbal IQ in children with autism spectrum disorders. Child Neuropsychol. 2006:12(4-5):247-67.

75. Hong MP, Eckert EM, Pedapati EV, Shaffer RC, Dominick KC, Wink LK, et al. Differentiating social preference and social anxiety phenotypes in fragile $X$ syndrome using an eye gaze analysis: a pilot study. J Neurodev Disord. 2019;11(1):1.

76. Mazzocco MM, Thompson L, Sudhalter V, Belser RC, Lesniak-Karpiak K, Ross JL. Language use in females with fragile $X$ or Turner syndrome during brief initial social Interactions. J Dev Behav Pediatr. 2006;27(4):319-28.

77. Turkstra LS, Abbeduto L, Meulenbroek P. Social cognition in adolescent girls with fragile x syndrome. Am J Intellect Dev Disabil. 2014;119(4):319-39.

78. Oakes A, Thurman AJ, McDuffie A, Bullard LM, Hagerman RJ, Abbeduto L. Characterising repetitive behaviours in young boys with fragile $\mathrm{X}$ syndrome. J Intellect Disabil Res. 2016;60(1):54-67.

79. Leekam SR, Prior MR, Uljarevic M. Restricted and repetitive behaviors in autism spectrum disorders: a review of research in the last decade. Psychol Bull. 2011;137(4):562-93.

80. Constantino JN. Social Responsiveness Scale. In: Volkmar FR, editor. Encyclopedia of autism spectrum disorders. New York: Springer New York; 2013. p. 2919-29.

81. McDuffie A, Abbeduto L, Lewis P, Kover S, Kim JS, Weber A, et al. Autism spectrum disorder in children and adolescents with fragile $X$ syndrome: within-syndrome differences and age-related changes. Am J Intellect Dev Disabil. 2010;115(4):307-26.

82. Luyster R, Gotham K, Guthrie W, Coffing M, Petrak R, Pierce K, et al. The Autism Diagnostic Observation Schedule-toddler module: a new module of a standardized diagnostic measure for autism spectrum disorders. J Autism Dev Disord. 2009;39(9):1305-20.

83. Skuse DH. Rethinking the nature of genetic vulnerability to autistic spectrum disorders. Trends Genet. 2007;23(8):387-95.

\section{Publisher's Note}

Springer Nature remains neutral with regard to jurisdictional claims in published maps and institutional affiliations.

Ready to submit your research? Choose BMC and benefit from:

- fast, convenient online submission

- thorough peer review by experienced researchers in your field

- rapid publication on acceptance

- support for research data, including large and complex data types

- gold Open Access which fosters wider collaboration and increased citations

- maximum visibility for your research: over $100 \mathrm{M}$ website views per year

At BMC, research is always in progress.

Learn more biomedcentral.com/submissions 